\title{
Small-cell lung carcinoma with long-term survival: A case report
}

\author{
KAZUMI NISHINO ${ }^{1}$, FUMIO IMAMURA ${ }^{1}$, TORU KUMAGAI ${ }^{1}$, JUNJI UCHIDA ${ }^{1}$, \\ YUKI AKAZAWA $^{1}$, TAKAKO OKUYAMA ${ }^{1}$ and YASUHIKO TOMITA ${ }^{2}$ \\ ${ }^{1}$ Department of Thoracic Oncology; ${ }^{2}$ Institute of Pathology, Osaka Medical Center for \\ Cancer and Cardiovascular Diseases, Osaka 537-8511, Japan
}

Received March 28, 2011; Accepted June 20, 2011

DOI: $10.3892 / \mathrm{ol} .2011 .355$

\begin{abstract}
Small-cell lung carcinoma is the most aggressive among lung cancer subtypes, has a poor prognosis and is highly associated with smoking. We present a case of small-cell lung carcinoma in a patient who had never smoked and has survived for 14 years without achieving a complete remission since the first relapse. His long-term survival may be ascribed to the slow growth of the cancer cells, limited metastasis and favorable responses to the treatments he has received. During these 14 years, only two lymph node metastases and a single metastasis to the brain developed. His small-cell lung carcinoma has been well controlled each time by the various treatments he has received, including chemotherapy, radiotherapy and surgery. Pathologically, the tumor was a typical small-cell lung carcinoma with extensive necrosis. Results showed the mitotic rate and the cell proliferation markers to be greater than those in the intermediate-grade atypical carcinoid, but relatively low. Thus, we conclude that this case belongs to an overlap between intermediate- and high-grade neuroendocrine tumors.
\end{abstract}

\section{Introduction}

The 2004 World Health Organization (WHO) classification proposed four subtypes of pulmonary neuroendocrine (NE) tumors: low-grade typical carcinoid (TC), intermediate-grade atypical carcinoid (AC) and two high-grade tumors, large cell neuroendocrine carcinoma (LCNEC) and small-cell lung carcinoma (SCLC) (1). SCLC is a highly aggressive cancer and results in mortality in 2-4 months without treatment. Most patients respond to primary therapy, but survival remains poor and median survival times are reported to be approximately 24 months in limited disease and 12 months in extensive

Correspondence to: Dr Kazumi Nishino, Department of Thoracic Oncology, Osaka Medical Center for Cancer and Cardiovascular Diseases, 1-3-3 Nakamichi, Higashinari-ku, Osaka 537-8511, Japan E-mail: nishinishipptruth@yahoo.co.jp

Key words: small-cell lung carcinoma, pulmonary neuroendocrine tumors, atypical carcinoid, The 2004 WHO Classification, long-term survival disease (2,3). In this study, we present a case of SCLC in a never smoker who has survived for 14 years without achieving a complete remission following the initial relapse.

\section{Case report}

In November 1996, a 44-year-old male, with no history of smoking, presented at the Osaka Medical Center for Cancer and Cardiovascular Diseases with an abnormal hilar shadow in the left lung, complaining of cough and dyspnea. A computerized tomography (CT) scan revealed a $4.5 \times 3.0 \mathrm{~cm}$ hilar mass in the left lung (Fig. 1A). The patient was cytologically diagnosed with SCLC by bronchoscopic examination (Fig. 1B). Metastatic workup demonstrated that he had limited disease, cT2aN2M0 stage IIIA (the 7th edition of the TNM system for lung cancer). The values of serum neuron-specific enolase and carcinoembryonic antigen were within normal limits and the pro-gastrin-releasing-peptide (ProGRP) was not measured at the time. The patient received four cycles of chemotherapy consisting of cisplatin (CDDP) and etoposide, with concurrent thoracic radiation of $44 \mathrm{~Gy}$ at $2.2 \mathrm{~Gy} /$ fraction daily. The treatment resulted in a complete response. Prophylactic cranial irradiation was not performed since there was no evidence to recommend it at the time (4).

The patient remained asymptomatic and no sign of disease recurrence was detected until December 1998, when right mandibular lymphadenopathy was evidenced. By that time, the level of ProGRP had gradually been elevating from $25 \mathrm{pg} /$ $\mathrm{ml}$ in October 1997 to 76 pg/ml in August 1998 and 133 pg/ $\mathrm{ml}$ in December 1998 (normal range 0-45 pg/ml). Aspiration needle cytology of the lymph node revealed metastasis of SCLC, leading to the diagnosis of recurrence of SCLC as the cancer cells obtained from the lymph node revealed almost the same morphological features as the primary lung tumor cells. Since imaging studies showed no recurrence with the exception of the lesion, and the WBC count was $\sim 3,000 / \mu 1$, the patient was administered palliative radiotherapy with a total dose of 70 Gy without chemotherapy. The lymphadenopathy disappeared and the level of ProGRP decreased to $14.1 \mathrm{pg} / \mathrm{ml}$. Two years later, in April 2000, the right axillary lymph node was found to be enlarged and cytology revealed metastasis of SCLC. Palliative radiotherapy with a total dose of 60 Gy was administered to the lesion. The lymph node swelling did not disappear completely, but the level of ProGRP decreased from 154 to $44 \mathrm{pg} / \mathrm{ml}$. Although the level of ProGRP was slowly 
A

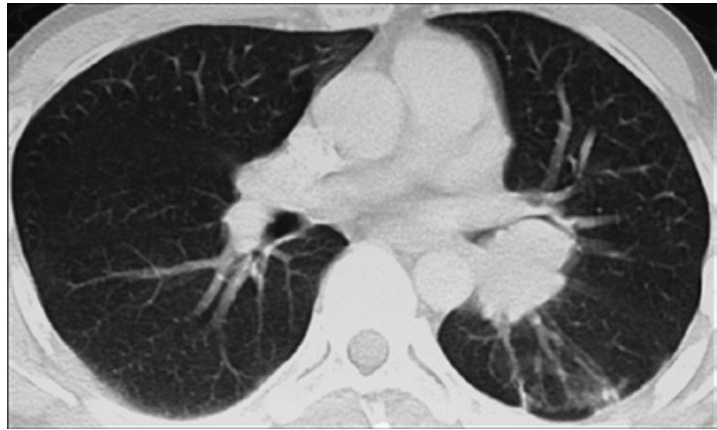

B

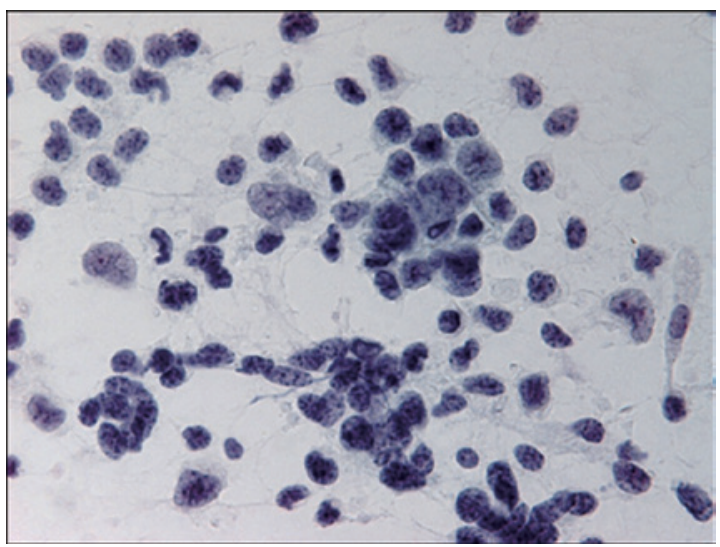

Figure 1. (A) A CT image in November 1996 revealed a 4.5x3.0-cm hilar mass in the left lung. (B) Cytology of the material obtained bronchoscopically from the primary lung tumor revealed SCLC.

elevated to $150 \mathrm{pg} / \mathrm{ml}$ in November 2002, the patient observed no further symptoms and subsequently stopped consultation with the hospital.

The patient presented at the Osaka Medical Center for Cancer and Cardiovascular Diseases again in September 2006. Neurological examinations at admission indicated cerebral abnormality: left upper 1/4 homonymous hemianopsia and dysrhythmia on the electroencephalogram. The level of ProGRP was markedly elevated $(2,860 \mathrm{pg} / \mathrm{ml})$. Magnetic resonance imaging (MRI) of the brain revealed a huge mass in the right temporal lobe (Fig. 2A). The brain tumor was completely excised and histopathological examination determined it to be a metastasis of SCLC. The tumor was cytologically identical to the primary lung cancer, showing extensive necrosis, a high nuclear-to-cytoplasmic ratio and fine nuclear chromatin. The mitotic rate was 14 mitoses per 10 high-power fields (HPF) in this resected specimen. The Ki-67 labeling index was $25 \%$. Immunohistochemical stains were positive for NE markers, including chromogranin A, synaptophysin and CD56 (Fig. 2B). The primary hilar tumor in the left lung and the right axillary lymph node revealed an increased uptake of fludeoxyglucose in positron emission tomography (PET) scanning. The patient received whole brain radiation therapy (WBRT) (30 Gy in 10 fractions), followed by systemic chemotherapy with CDDP and irinotecan hydrochloride (CPT11). Although the doses of CDDP and CPT11 were reduced to 50 and $50 \mathrm{mg} / \mathrm{m}^{2}$, respectively, ProGRP levels decreased notably to $90.7 \mathrm{pg} / \mathrm{ml}$, following chemotherapy.

From September 2007, the level of ProGRP was again gradually elevated. Recurrence of brain metastasis was
A

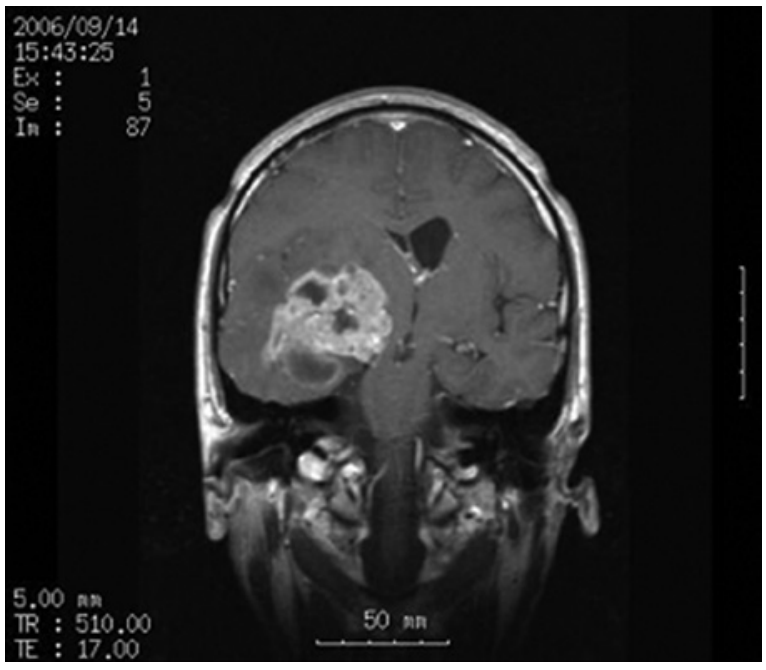

B

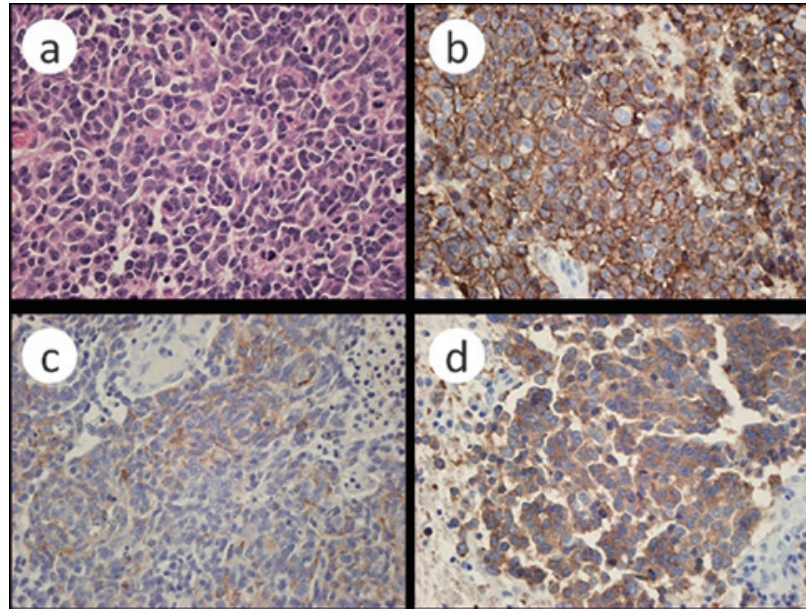

Figure 2. (A) Brain MRI in September 2006 showed a huge mass in the right temporal lobe. (B) Hematoxylin and eosin (H\&E) and immunohistological staining of the specimen from the resected brain tumor. The tumor cells were positive for CD56, chromogranin and synaptophysin, indicating the neuroendocrine origin of the tumor. a, H\&E stain; b, CD56 stain; c, chromogranin stain; d, synaptophysin stain. Original magnification, x200.

detected on the MRI in November 2008 and the patient underwent intensity-modulated radiotherapy (IMRT) for the brain tumor. Following IMRT, the patient was administered chemotherapy with CDDP and CPT11. However, compliance to the chemotherapy was poor due to hematological toxicity. In September 2009, the patient was admitted for obstructive pneumonia in the left lower lobe with high fever, and treated successfully with antibiotics. The level of ProGRP elevated to $724 \mathrm{pg} / \mathrm{ml}$ and distinct progression of the primary hilar tumor in the left lung was again detected by CT. The patient refused to complete systemic chemotherapy and was followed up for 1 year. In November 2010, CT and PET detected distinct progression of the primary lung tumor resulting in atelectasis of the left lower lobe and right axillary lymphadenopathy. The level of ProGRP was elevated to $1,640 \mathrm{pg} / \mathrm{ml}$. Chemotherapy with amrubicin was administered in December 2010.

At present, the clinical course of the patient has continued for 14 years following the initial diagnosis of SCLC and 4 years following the diagnosis of brain metastasis. The brain remains relapse-free at present. The patient is currently 
continuing treatment with amrubicin for SCLC and his performance remains positive.

\section{Discussion}

NE tumors represent approximately $20 \%$ of all primary lung neoplasms (5). NE tumors of the lung are separated into four subgroups: low-grade TC, intermediate-grade AC and two high-grade malignancies, LCNEC and SCLC, according to WHO in 2004 (1). SCLC is the most common NE tumor (20\% of total lung cancers), followed by LCNEC (3\%), TC $(2 \%)$ and $\mathrm{AC}(0.2 \%)(6)$. The tumors differ morphologically, immunohistochemically and structurally. The WHO classification defines SCLC as a NE tumor with greater than 10 mitoses/10 HPF and small-cell cytologic features. TC is considered a NE tumor with carcinoid morphology, fewer than 2 mitoses/10 HPF and lacking in necrosis, while $\mathrm{AC}$ is defined as a NE tumor with carcinoid morphology showing 2-10 mitoses/10 HPF or necrosis (1). The grade of malignancy of each NE subtype is correlated with clinicopathological behavior and prognosis of the disease. TC and AC are relatively slow-growing tumors and generally exhibit a favorable outcome, while LCNEC and SCLC are very aggressive with a dismal prognosis $(5,6)$.

The accurate differential diagnosis of carcinoids from SCLC is critical in the selection of the appropriate treatment. Usually, SCLC is rarely mistaken for carcinoids, with the exception of small biopsy materials. There are also certain differences in the clinical background and profiles according to the subgroup of NE tumors. Unlike carcinoids, SCLC is markedly associated with a history of smoking $(7,8)$. Carcinoids tend to occur in younger patients (mean age 45-50 years), whereas the high-grade NE tumors affect older patients (mean age 65 years). The former are capable of distant metastases in less than $20 \%$ of cases (most commonly to liver and bones), and SCLC tends to metastasize to the brain, liver, adrenal glands and bone with higher frequency $(5,6)$. Due to the low response rates for chemo- and radiotherapy, surgical resection is primarily used in the treatment of carcinoids, whereas the standard treatment for limited-stage SCLC includes combined chemoradiotherapy due to high sensitivity.

This case was initially diagnosed as SCLC in 1996 by cytological sampling obtained using bronchoscopy. The initial chemoradiotherapy resulted in a complete response. Ten years later, a metastatic brain tumor was excised. Although the clinical course was not typical for SCLC, the histopathological features of the resected tumor confirmed the diagnosis of SCLC due to the morphology of the tumor cells, the positive staining with neuroendocrine markers and the 14 mitoses/10 HPF with extensive necrosis (according to the WHO classification in 2004). The Ki-67 proliferative index has recently been considered to be useful in distinguishing between the various subtypes of NE tumors, particularly in small biopsy and cytology specimens. The Ki-67 proliferation rate of TC is less than 2\% and AC is less than 20\% (typical rate $\sim 10 \%$ ), while the two high-grade NE tumors are higher than $20 \%$ (typical rate for SCLC is $60-100 \%)(6,9,10)$. The Ki-67 index of this case was $25 \%$.

The clinical manifestations of this case, such as slowgrowing tumors, limited metastatic potential and a favorable prognosis, with an over 14-year survival, support the diagnosis of AC, while the morphological, immunohistochemical and structural features of the tumors are typical of SCLC. We believe that this case fits the diagnostic criteria of SCLC according to the WHO classification, but it is a borderline case between AC and SCLC. Asamura et al reported that 5-year survival rates for TC, AC, LCNEC and SCLC in Japanese surgical cases of NE tumors were 96.2, 77.8, 40.3 and 35.7\%, respectively (8). An analysis of Japanese lung cancer patients registered in 2002 revealed that SCLC accounted for $9.2 \%$ of new lung cancer cases in Japan, and 5-year survival rates were $17.2 \%$ for stage IIIA, $12.4 \%$ for stage IIIB, $3.8 \%$ for stage IV and $14.7 \%$ overall (11).

The prognosis is particularly dismal in SCLC patients with brain metastasis. In the practice guidelines recently published in the Journal of Neurooncology, the authors recommend surgical resection followed by WBRT for newly-diagnosed single brain metastases, which improves outcomes when compared to WBRT alone. However, these authors indicate that the recommendation does not apply to relatively radiosensitive tumors such as SCLC (12). By contrast, JesienLewandowicz et al assert that patients with solitary brain metastasis from SCLC should be treated radically, in particular those at younger ages with a small primary tumor in the lung, good performance status and lack of systemic dissemination (13). Four case reports describe excellent long-term survival following resection of a solitary metastatic brain tumor of SCLC and adjuvant WBRT (13-16). In the present case, surgical resection followed by WBRT and chemotherapy was successful. Imai et al suggest that a subtype of slow-growing SCLC, which shows different biological properties, should be distinguished from the common type SCLC (16). Although unusual, patients with this subtype of NE tumor may potentially achieve longer survival than those with typical SCLC, and should be treated with local and multimodality treatment on a case-by-case basis.

In conclusion, we present a case report of a SCLC patient who has survived for 14 years following initial diagnosis with persistent disease, in spite of repetitive multimodality therapies. This case suggests the existence of borderline cases between intermediate- and high-grade NE tumors, and that long-term survival may be expected with suitable treatments. A method should be established to select SCLC patients with a favorable prognosis, such as this case, and to find optimal therapeutic approaches for such patients.

\section{References}

1. Beasley MB, Brambilla E and Travis WD: The 2004 World Health Organization classification of lung tumors. Semin Roentgenol 40: 90-97, 2005.

2. Jackman DM and Johnson BE: Small-cell lung cancer. Lancet 366: 1385-1396, 2005.

3. El Maalouf G, Rodier JM, Faivre S and Raymond E: Could we expect to improve survival in small cell lung cancer? Lung Cancer 57 (Suppl 2): 30-34, 2007.

4. Auperin A, Arriagada R, Pignon JP, et al: Prophylactic cranial irradiation for patients with small-cell lung cancer in complete remission. Prophylactic cranial irradiation overview collaborative group. N Engl J Med 341: 476-484, 1999.

5. Gustafsson BI, Kidd M, Chan A, Malfertheiner MV and Modlin IM: Bronchopulmonary neuroendocrine tumors. Cancer 113: 5-21, 2008. 
6. Rekhtman N: Neuroendocrine tumors of the lung: an update. Arch Pathol Lab Med 134: 1628-1638, 2010.

7. Ou SH, Ziogas A and Zell JA: Prognostic factors for survival in extensive stage small cell lung cancer (ed-sclc): the importance of smoking history, socioeconomic and marital statuses, and ethnicity. J Thorac Oncol 4: 37-43, 2009.

8. Asamura H, Kameya T, Matsuno Y, et al: Neuroendocrine neoplasms of the lung: a prognostic spectrum. J Clin Oncol 24: $70-76,2006$

9. Aslan DL, Gulbahce HE, Pambuccian SE, Manivel JC and Jessurun J: Ki-67 immunoreactivity in the differential diagnosis of pulmonary neuroendocrine neoplasms in specimens with extensive crush artifact. Am J Clin Pathol 123: 874-878, 2005.

10. Pelosi G, Rodriguez J, Viale G and Rosai J: Typical and atypical pulmonary carcinoid tumor overdiagnosed as small-cel carcinoma on biopsy specimens: a major pitfall in the management of lung cancer patients. Am J Surg Pathol 29: 179-187, 2005

11. Sawabata N, Asamura H, Goya T, Mori M, Nakanishi Y, Eguchi K, Koshiishi Y, Okumura M, Miyaoka E and Fujii Y: Japanese lung cancer registry study: first prospective enrollment of a large number of surgical and nonsurgical cases in 2002 J Thorac Oncol 5: 1369-1375, 2010.
12. Gaspar LE, Mehta MP, Patchell RA, et al: The role of whole brain radiation therapy in the management of newly diagnosed brain metastases: a systematic review and evidence-based clinical practice guideline. J Neurooncol 96: 17-32, 2010.

13. Jesien-Lewandowicz E, Spych M, Fijuth J and Kordek R: Solitary brain metastasis of an occult and stable small-cell lung cancer in a schizophrenic patient: a 3-year control. Lung Cancer 69: 245-248, 2010.

14. Abratt RP, de Groot M and Willcox PA: Resection of a solitary brain metastasis in a patient with small cell lung cancer long-term survival. Eur J Cancer 31A: 419, 1995.

15. Harrison ML and Goldstein D: Prolonged survival in a patient with an occult primary small-cell lung cancer and a solitary brain metastasis at diagnosis. Intern Med J 32: 621-622, 2002.

16. Imai R, Hayakawa K, Sakurai H, Nakayama Y, Mitsuhashi N and Niibe H: Small cell lung cancer with a brain metastasis controlled for 5 years: a case report. Jpn J Clin Oncol 31: 116-118, 2001 